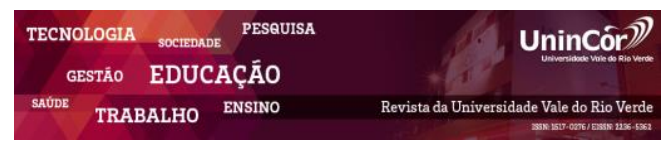

Revista da Universidade Vale do Rio Verde ISSN: 1517-0276 / EISSN: 2236-5362 v. $17 \mid$ n. 1 | Ano 2019

Richard da Silva Pereira Calazans Centro Universitário Luterano de Ji-Paraná E-mail: richardccalazans@hotmail.com

Alexandra Luiza Silva Bulian
Centro Universitário Luterano de Ji-Paraná
E-mail: alebulian@ @otmail.com

Lorraynie Oliveira Alves Centro Universitário Luterano de Ji-Paraná E-mail: lo.alves@outlook.com

Karine Amanda Costa Centro Universitário Luterano de Ji-Paraná E-mail: karineamanda@live.com.pt

Jeferson de Oliveira Salvi Centro Universitário Luterano de Ji-Paraná E-mail: jefersonsalvi@ hotmail.com

\section{ESTUDO FITOQUÍMICO E AVALIAÇÃO \\ DA CITOTOXICIDADE AGUDA FRENTE À ARTEMIA SALINA (LEACH) DE PLANTAS COMERCIALIZADAS EM FEIRA-LIVRE}

\section{RESUMO}

A utilização de plantas medicinais como recurso terapêutico constitui uma prática historicamente difundida e muito comum. As espécies Morus nigra, Morus alba, Cordia ecalyculata e Anadenanthera macrocarpa, popularmente conhecidas como amoreira-negra, amoreira-branca, folia-magra e angico, respectivamente, são amplamente utilizadas pela população brasileira para diversos fins. O presente estudo objetivou realizar o screening fitoquímico e avaliar a citotoxicidade aguda das referidas espécies. Realizou-se a triagem fitoquímica através de testes qualitativos, utilizando-se extratos aquosos. A citotoxicidade foi avaliada por meio da determinação da $\mathrm{DL}_{50}$ frente à Artemia salina, submetendo-se 10 náuplios a extratos aquosos nas concentrações de $16,3 \mathrm{mg} / \mathrm{mL} ; 8,15 \mathrm{mg} / \mathrm{mL} ; 4,075$ $\mathrm{mg} / \mathrm{mL} ; 2,0375 \mathrm{mg} / \mathrm{mL}$ e $1,0187 \mathrm{mg} / \mathrm{mL}$. O bioensaio foi realizado em triplicata e a mortalidade avaliada após $24 \mathrm{~h}$. A triagem fitoquímica revelou a presença de cumarinas, flavonoides e taninos condensados nas amoreiras e folia-magra, estando presente também nesta última triterpenos/esteroides. No angico, revelou-se a presença de antraquinonas, saponinas, taninos condensados e triterpenos/esteroides. No teste de citotoxicidade, encontrou-se $\mathrm{DL}_{50}$ de $2.170 \mu \mathrm{g} / \mathrm{mL}$ para a amoreira-negra, $2.243 \mu \mathrm{g} / \mathrm{mL}$ para a amoreira-branca, 1.805,9 $\mu \mathrm{g} / \mathrm{mL}$ para a folia-magra e de $6.401 \mu \mathrm{g} / \mathrm{mL}$ para o angico. Os extratos foram classificados como atóxicos mediante os critérios estabelecidos por Meyer e colaboradores (1982). Identificou-se a presença de diferentes metabólitos secundários associados à algumas das atividades terapêuticas relatadas na literatura. Sugere-se a investigação do potencial biológico das espécies em questão, bem como, a pesquisa sobre a genotoxicidade e a mutagenicidade de modo a contribuir com o uso racional das plantas investigadas.

Palavras-chave: Citotoxicidade. Prospecção-Fitoquímica. Artêmia-salina. Fitoterapia. Plantas-Medicinais.

\section{PHYTOCHEMICAL STUDY AND EVALUATION OF ACUTE CYTO- TOXICITY AGAINST THE SALINE ARTEMIA (LEACH) OF PLANTS MARKETED IN FAIR-FREE}

\title{
ABSTRACT
}

The use of medicinal plants as a therapeutic resource is a historically widespread and very common practice. The species Morus nigra, Morus alba, Cordia ecalyculata and 
Anadenanthera macrocarpa, popularly known as amoreiranegra, amoreira-branca, folia-magra and angico, respectively, are widely used by the Brazilian population for various purposes. The present study aimed to perform the phytochemical screening and to evaluate the acute cytotoxicity of these species. Phytochemical screening was carried out through qualitative tests using aqueous extracts. Cytotoxicity was assessed by the determination of LD50 against Artemia salina, subjecting 10 nauplii to aqueous extracts at concentrations of $16.3 \mathrm{mg} / \mathrm{mL}$; $8.15 \mathrm{mg} / \mathrm{mL} ; 4.075 \mathrm{mg} / \mathrm{mL} ; 2.0375 \mathrm{mg} / \mathrm{mL}$ and $1.0187 \mathrm{mg} / \mathrm{mL}$. The bioassay was performed in triplicate and the mortality evaluated after 24 hours. Phytochemical screening revealed the presence of coumarins, flavonoids and condensed tannins in amoreiras and folia-magra, being also present in this last triterpenos / steroids. In the angico, the presence of anthraquinones, saponins, condensed tannins and triterpenes / steroids were revealed. In the cytotoxicity test, LD 50 of $2,170 \mu \mathrm{g}$ / $\mathrm{mL}$ was found for amoreira negra, 2,243 $\mu \mathrm{g} / \mathrm{mL}$ for amoreira branca, $1,805.9 \mu \mathrm{g} / \mathrm{mL}$ for folia magra, and $6,401 \mu \mathrm{g} / \mathrm{mL}$ for angico. The extracts were classified as nontoxic using the criteria established by Meyer et al. (1982). It was identified the presence of different secondary metabolites associated to some of the therapeutic activities reported in the literature. It is suggested to investigate the biological potential of the species in question, as well as research on genotoxicity and mutagenicity in order to contribute to the rational use of the investigated plants.

Keywords: Cytotoxicity. Screnning Phytochemical. Artemia salina. Phytotherapy. Medicinal plants.

Recebido em: 02/09/2018 - Aprovado em: 03/04/2019 - Disponibilizado em: 15/07/2019

\section{INTRODUÇÂO}

Com a ascendência dos processos de urbanização e industrialização, a utilização de plantas medicinais passou por um declínio devido ao crescimento da oferta e do consequente consumo de medicamentos sintéticos. Nesse cenário, houve a contenção das pesquisas envolvendo a caracterização dos fitoquímicos, bem como, a redução dos estudos que buscassem investigar os possíveis efeitos biológicos a eles associados (LORENZI; MATOS, 2008; LÓS et al., 2012). No entanto, nas últimas décadas, se observa o aumento da exploração do potencial biológico das plantas medicinais, no Brasil e no mundo, impulsionado pelas novas tecnologias aplicadas à descoberta de novos compostos nos diferentes estudos etnobotânicos e farmacobotânicos (FREITAS et al., 2012; ALVES et al., 2016).

O interesse no desenvolvimento de opções terapêuticas eficientes, baseadas na medicina popular, é sustentado pela vigente carência dos órgãos públicos de saúde, e a dificuldade que estes enfrentam em custear a aquisição dos diferentes fármacos que compõem o arsenal terapêutico (PARENTE; ROSA, 2001; FREITAS et al, 2012).

Além disso, é importante se considerar o saber cultural que se materializa no hábito da aquisição dos diferentes preparados das espécies vegetais, disponíveis na flora brasileira e 
comercializados em feiras livres, como o responsável por preservar e difundir opções terapêuticas que foram transmitidas entre as diferentes gerações ao longo do tempo (MAIOLIAZVEDO; $\quad$ FONSECA-KRUEL, 2007; MONTEIRO et al., 2010).

A $M$. nigra e a $M$. alba pertencem à família Moraceae e são popularmente conhecidas como amoreira-negra e amoreira-branca, respectivamente. São nativas da Ásia, África e América do Norte, mas também podem ser encontradas em outras regiões do mundo, incluindo o Brasil (WASANO et al., 2009). As indicações medicinais destas plantas incluem a redução do índice glicêmico, dos níveis do colesterol e dos sintomas característicos da menopausa (VENDRUSCOLO; MENTZ, 2006), bem como, apresentam efeitos sedativos, expectorantes, diuréticos, laxativos, antiepiléticos, analgésicos, anti-inflamatórios, antioxidantes, anti-helmínticos, dentre outros (ERCISLI; ORHAN, 2007; CHEN et al., 2013).

Folia-magra, Porangaba e Chá-de-bugre são três diferentes denominações populares para a espécie Cordia ecalyculata Vell. (sinonímia de Cordia salicifolia Cham.). Pertencente à família Boraginaceae, pode ser encontrada em florestas tropicais da Argentina e do Paraguai, como também em diferentes regiões do Brasil (LORENZI, 2002; BARROSO et al., 2002). A $C$. ecalyculata é empregada como agente emagrecedor e diurético, utilizada no tratamento de úlceras gástricas, úlceras externas (em forma de banho), tosses catarrais, reumatismo, picada de cobra, como agente anti-inflamatório, tônico, hipolipidêmico e redutor de colesterol, cardiotônico e energético (BARROSO et al., 2002; ARREBOLA et al, 2004; DIAS, 2004; BARBOSA-JUNIOR，2005; DUARTE，2006; PELIZZA, 2010; PIZZIOLO et al, 2011).

A espécie Anadenanthera macrocarpa, por sua vez, pertence à subfamília Mimosoideae, da família Leguminosae. É conhecida pela denominação de angico ou angico vermelho, sendo nativa das florestas tropicais da América do Sul, pode ser encontrada em várias regiões do Brasil (CARVALHO, 1994; SILVA, 2011). Dentre as indicações terapêuticas da $A$. macrocarpa estão incluídas: o tratamento de inflamações, bronquite, tosse, coqueluche, aftas, febre, disenteria, helmintoses e dores nas pernas (MATOS, 1997; DESMARCHELIER et al., 1999; MUNÕZ, et al, 2000; FIGUEREDO et al., 2013; RODRIGUES, et al., 2014).

O presente estudo teve o intuito de identificar os metabólitos secundários presentes nos infusos obtidos das plantas Morus nigra, Morus alba, Cordia ecalyculata e Anadenanthera macrocarpa, bem como, objetivou avaliar a citotoxidade aguda mediante a determinação da dose letal mediana (DL50).

\section{METODOLOGIA}

Os materiais botânicos foram adquiridos em feira livre, no mercado público de Porto Alegre, estado do Rio Grande do Sul. Como critérios para a seleção e aquisição dos exemplares, se considerou as espécies mais comercializadas na época, sendo que, as embalagens estavam devidamente íntegras e identificadas pelos seus respectivos nomes científicos e partes vegetais. As amostras, 
portanto, corresponderam às folhas de Morus alba, Morus nigra e Cordia ecalyculata e às cascas de troncos de Anadenanthera macrocarpa.

As amostras foram transportadas e os experimentos foram desenvolvidos no laboratório de fitoquímica do Centro Universitário Luterano de Ji-Paraná, estado de Rondônia. Após a inspeção da integridade das embalagens, foram produzidas das infusões das folhas e o decocto da A. macrocarpa.

Para a prospecção fitoquímica padronizou-se a utilização de $15 \mathrm{~g}$ das amostras para cada $150 \mathrm{~mL}$ de água destilada, resultando na concentração de $100 \mathrm{mg} / \mathrm{mL}$ (DICKEL et al., 2010).

No teste de citotoxicidade aguda a concentração inicial baseou-se no relato popular do uso de duas colheres de sopa do material vegetal para uma xícara de chá $(240 \mathrm{~mL})$, o que estabeleceu a padronização da concentração de $16,3 \mathrm{mg} / \mathrm{mL}$ e, em seguida, se realizou consecutivas diluições seriadas (1:2, 1:4, 1:8, $1: 16)$.

\subsection{Prospecção Fitoquímica}

A realização de triagem fitoquímica a partir dos extratos aquosos das folhas de $M$. nigra, M. alba, Cordia ecalyculata e cascas do tronco de Anadenanthera macrocarpa ocorreu fundamentada a a partir de testes qualitativos baseados no princípio de colorimetria e precipitação de compostos, seguindo metodologias reconhecidas. Os compostos pesquisados foram: Flavonoides, Taninos, Saponinas, Cumarinas (RADI; TERRONES, 2007), Antraquinonas (RODRIGUES et al.,
2009), Purinas e Triterpenoides/esteróides (TEIXEIRA et al., 2012).

\subsection{Citotoxicidade Aguda}

Os náuplios do microcrustáceo foram obtidos após a eclosão em solução de sal marinho (pH 8,5), sob controle de temperatura $25^{\circ} \mathrm{C}( \pm 2)$, incidência de luz de (100 w) e aeração, por 48 horas.

Todas as concentrações preparadas para cada um dos extratos aquosos das espécies vegetais foram testadas em triplicatas, a partir da adição de 10 náuplios de $A$. salina à $5 \mathrm{~mL}$ às diferentes diluições. Como controle negativo se utilizou apenas a solução marinha, seguindo a metodologia proposta por Meyer e colaboradores (1982).

Decorridas 24 horas, se realizou a contagem dos sobreviventes e calculou-se o percentual de mortalidades $(\% \mathrm{M})$, conforme a fórmula que segue:

$$
\% \mathrm{M}=100-(\mathrm{MIVx100/MIVen})
$$

*MIV = média dos indivíduos vivos por concentração; **MIVcn = Média dos indivíduos vivos do controle negativo.

A determinação da dose letal mediana (DL50) foi realizada por meio do método de regressão linear considerando a equação da reta obtida pela relação logarítma das concentrações com as suas respectivas mortalidades.

Considerando ainda a sobrevivência dos náuplios, determinou-se a análise da variância seguida pelo teste de Dunnet para múltiplas comparações de cada extrato em relação ao controle, com o auxílio do software Graphpad Prism (Versão 5.0). 


\section{RESULTADOS E DISCUSSÃO}

Com a realização da triagem fitoquímica foi possível constatar a presença ou ausência de compostos pertencentes às classes de metabólitos secundários (taninos, flavonoides, esteroides, triterpenos, saponinas, antraquinonas, purinas e cumarinas) na análise das quatro plantas. A tabela 1 exibe os resultados obtidos na triagem fitoquímica de cada espécie vegetal testada.

Tabela 1 - Resultados do screening fitoquímico realizado a partir das infusões das folhas de M. alba, M. nigra, C. ecalyculata e decocto das cascas do tronco de A. macrocarpa.

\begin{tabular}{lcccc}
\hline Metabólitos secundários & M. alba & M. nigra & C. ecalyculata & A. macrocarpa \\
\hline Antraquinonas & - & - & - & + \\
Cumarinas & + & + & + & - \\
Flavonoides & + & + & + & - \\
Purinas & - & - & - & - \\
Saponinas & - & - & - & + \\
Taninos Condensados & + & + & + & + \\
Taninos Hidrolisáveis & - & - & - & - \\
Triterpernos/Esteroides & - & - & + & + \\
\hline
\end{tabular}
Legenda: "+" = Positivo ou Presente. "-“= Negativo ou Ausente. Fonte: Autores.

$\mathrm{Na}$ análise fitoquímica de ambas as amoreiras, foram detectadas as mesmas classes de metabólitos secundários: cumarinas, flavonoides e taninos condensados. Em triagem fitoquímica realizada nas folhas de $M$. nigra por Lima e Cunha (2018) determinou-se a presença de cumarinas, chalconas e auronas, bem como, a de terpenoides e esteroides, por Padilha e colaboradores (2010), na mesma parte vegetal. Ainda, foram relatadas a presença de saponinas, taninos e flavonoides nas folhas de $M$. alba por Yadav \& Nade (2008) e Wang e colaboradores (2008).

Diversos compostos fitoquímicos são encontrados em plantas do gênero Morus sp., como o alcaloide moranolina e glicoproteínas, associados ao efeito hipoglicemiante, além da desoxinojirimicina um potente inibidor da alfaglicosidade, em raízes e folhas (KIM et al., 1999). Além disso há registros da presença de compostos fenólicos, incluindo flavonoides isoprenilados, estilbenos, 2-arilbenzopiranos, cumarinas, cromonas, xantonas e outros (NOMURA et al., 1988; NOMURA; HANO, 1994).

Conforme os achados no presente estudo, para a $C$. ecalyculata, compostos da classe dos alcaloides também são relatados em pesquisas, as quais incluem a cafeína, o ácido alantoico, a alantoína, bem como, glicosídeos como a consolidina, taninos e pigmentos (ASSONUMA, 2009). Arrebola e colaboradores (2004) ressaltam, ainda, que algumas dessas substâncias são encontradas na família Mimosoideae como um todo, tais como: os alcaloides, as quinonas, saponinas, taninos, ácidos fenólicos, mucilagens, polissacarídeos, flavonoides e ácidos graxos de interesse terapêutico e nutricional, como o ácido gamalinolênico.

Já na análise das cascas do tronco de $A$. macrocarpa ou angico vermelho, evidenciou-se a presença de compostos das classes antraquinonas, saponinas, taninos condensados e 
triterpenoides/esteroides. Tal espécie é amplamente conhecida por ser rica em taninos justamente nesta parte da planta. No entanto, outros compostos evidenciados também já foram relatados, incluindo triterpenoides (em especial a luperona e lupeol), alcaloides, flavonoides e fenóis (dalbergina; 3,4,5- dimetoxidalbergiona) (DUKE, 1985; SILVA, 2011).

A variação dos resultados encontrada na triagem fitoquímica, realizada nesta pesquisa e corroborada por outros trabalhos, pode ser explicada conforme descrevem Simões e colaboradores (2000), os quais mencionam que a presença de metabólitos secundários em plantas é dependente de diversos fatores, tais como: a sazonalidade, época do ano e métodos de coleta, nível de estímulo externo para a síntese e a qualidade do solo. Essas condições influenciam na oscilação desses fitoquímicos de um espécime para outro, uma vez que, tais compostos não são distribuídos de modo homogêneo dentro do mesmo organismo vegetal.

Após a contagem dos sobreviventes, calculou-se o percentual médio das mortalidades de Artemia salina nas diferentes concentrações testadas para cada infuso preparado, conforme demonstrado na Tabela 2.

Tabela 2 - Percentual Médio de Mortalidade de $A$. salina em infusões de $M$. alba, M. nigra, $C$. ecalyculata e $A$. macrocarpa em diferentes concentrações.

\begin{tabular}{ccccc}
\hline \multicolumn{5}{c}{ Percentual Médio de Mortalidade } \\
\hline $\begin{array}{c}\text { [ ] } \\
\mathbf{m g} / \mathbf{m L}\end{array}$ & M. alba & M. nigra & $\begin{array}{c}\boldsymbol{C} . \\
\text { ecalyculat } \\
\boldsymbol{a}\end{array}$ & $\begin{array}{c}\boldsymbol{A} . \\
\text { macrocarpa }\end{array}$ \\
\hline 16,3 & $100 \%$ & $100 \%$ & $100 \%$ & $100 \%$ \\
8,150 & $80 \%$ & $100 \%$ & $100 \%$ & $60 \%$ \\
4,075 & $63,33 \%$ & $73,33 \%$ & $80 \%$ & $16,67 \%$ \\
2,037 & $50 \%$ & $40 \%$ & $64,44 \%$ & $13,33 \%$
\end{tabular}

$\begin{array}{ccccc}1,018 & 40 \% & 33,33 \% & 36,66 \% & 6,67 \% \\ \mathrm{CN} & 0 \% & 0 \% & 0 \% & 0 \%\end{array}$

Legenda: "[ ]" = Concentrações testadas. "CN" = Controle Negativo. Fonte: Autores.

$\mathrm{Na}$ determinação da dose letal mediana $\left(\mathrm{DL}_{50}\right)$ foram encontrados os valores de 2.243 $\mu \mathrm{g} / \mathrm{mL}$ para a $M$. alba, $2.170 \mu \mathrm{g} / \mathrm{mL}$ para a $M$. nigra, $1.805,9 \mu \mathrm{g} / \mathrm{mL}$ para a $C$. ecalyculata e $6.401 \mu \mathrm{g} / \mathrm{mL}$ para a A. macrocarpa.

Conforme o descrito por Meyer e colaboradores (1982), os extratos que apresentaram valores de $\mathrm{DL}_{50}$ superior a $1000 \mu \mathrm{g} / \mathrm{mL}$ são considerados atóxicos, ao passo que os que apresentarem valor inferior a $1000 \mu \mathrm{g} / \mathrm{mL}$ são considerados potencialmente tóxicos. Logo, seguindo estes parâmetros, todas as quatro espécies demonstraram-se atóxicas frente ao microcrustáceo Artemia salina L., visto que, ambas apresentaram valores de $\mathrm{DL}_{50}$ acima do valor pré-estabelecido.

Extratos etanólicos brutos das folhas de Morus nigra também foram avaliados frente ao microcrustáceo Artemia salina Leach por Lima e Cunha (2018), onde obteve-se um resultado de $\mathrm{DL}_{50}$ superior a $1000 \mu \mathrm{g} / \mathrm{mL}$, classificando-os, portanto, como atóxicos frente às larvas. Oliveira e colaboradores (2013) avaliaram a toxicidade subcrítica do extrato aquoso da amoreira-negra em ratos, determinando que o extrato pode ser considerado de baixa toxicidade, visto que não produziu efeitos tóxicos nos animais tratados.

Além disso, Castro (2010) avaliou a atividade estrogênica de extratos hidroalcóolicos das folhas de Morus nigra em ratas Wistar, demonstrando que o tratamento crônico com o extrato nas doses avaliadas não apresentou toxicidade. 
A citotoxicidade das folhas de Morus nigra L. também fora avaliada sobre linhagens celulares humanas, de hepatoma (HepG2), queratinóticos (HaCat) e fibroblastos humanos (HDFa), conclui-se que o extrato pode ser considerado seguro mediante a toxicidade celular (ALMEIDA-CINCOTTO et al., 2016).

Os resultados que caracterizam as infusões das folhas de $C$. ecalyculata como atóxicos corroboram com o encontrado nos estudos de Silva e colaboradores (2010), os quais investigaram o potencial genotóxico e citotóxico de extratos da mesma planta em camundongos suíços, constatando a ausência dos mesmos.

Em estudo similar desenvolvido por Luna e colaboradores (2005), a partir de extratos etanólicos das cascas de A. macrocarpa, constatou-se a indução de $80 \%$ de mortalidade dos náuplios de Artemia salina a $1000 \mu \mathrm{g} / \mathrm{mL}$, onde também se demonstrou um considerável efeito citotóxico. Toxicidade também foi observada por Muñoz e colaboradores (2000), exibindo relevante atividade in vivo. Tais evidências contrariam os resultados observados nesta pesquisa, provavelmente devido à maior concentração utilizada e de diferentes metabólitos extraídos da planta pela utilização de outro solvente.

O potencial biológico das plantas testadas pode ser avaliado mediante o uso racional das mesmas nos diferentes sistemas orgânicos, Carvalhinho e colaboradores (2017), por exemplo, concluíram sobre a eficácia da $A$. macrocarpa contra lepidópteros, destacando-a no manejo de pragas.
A determinação de padrões de segurança para o uso terapêutico de plantas necessita de estudos, in vitro e in vivo, que caracterizem a relação dose efeito dos compostos fitoquímicos das diferentes partes vegetais (Barcelos et al., 2017). Bem como no estabelecimento de concentrações médias de metabólitos ativos encontrados nas diferentes espécies, métodos de extração, posologias e indicações (VALIATTI, et. Al., 2018).

\section{CONCLUSÃO}

No presente estudo foi possível realizar a prospecção fitoquímica das plantas Morus nigra, Morus alba, Cordia ecalyculata e Anadenanthera macrocarpa, na qual foram evidenciados compostos derivados do metabolismo secundário dos vegetais, os quais são conhecidos por exercerem diversas atividades biológicas, justificando grande parte do potencial terapêutico de plantas medicinais. Atrelado a estes fatos, a partir do teste de citotoxicidade aguda frente o sistema de microcrustáceos Artêmia salina L., as amostras vegetais demonstraram baixa toxicidade ou até mesmo como atóxicas.

\section{REFERÊNCIAS}

1. ALMEIDA-CINCOTTO, M. G. J. et al. Avaliação do potencial citotóxico e do potencial em estimular a biossíntese de colágeno de extrato de folhas de Morus nigra $L$. em cultura celular. Rev. Ciên. Farm. Básica Apl., v. 37, n. 1, 2016.

2. ALVES, C. A. B. et al. Comercialização de plantas medicinais: um estudo etnobotânico na feira livre do município de Guarabira, Paraíba, nordeste do Brasil. Gaia Scientia, v. 10, ed. 4, p. 390-407. 2016. 
3. ARREBOLA, M.R.B. et al. Estudo dos componentes lipídicos das sementes de três espécies do gênero Cordia L. (Boraginaceae). Rev. Bras. Farmacogn., v. 14, n. 1, jan.-jun. 2004.

4. ASSONUMA, M. M. Determinação de alantoína e avaliação farmacológica de Cordia ecalyculata VELL (chá de bugre). 79 f. Dissertação (mestrado) - Universidade Estadual Paulista, Instituro de Química, 2009.

5. BARBOSA JÚNIOR, A. Plantas medicinais: descubra o que os vegetais podem fazer pela sua saúde. São Paulo: Universo dos Livros, 126p. 2005.

6. BARROSO, I.C.E. et al. O gênero Cordia L.: botânica, química e farmacologia. Revista Lecta, v.20, n.1, p.15-34, 2002.

7. CAMPOS, S.C. et al. Toxicidade de espécies vegetais. Revista Brasileira de Plantas Medicinais, v.18, n.1, supl. I, p.373-382, 2016.

8. CARVALHINHO, D.T. et al. Toxicidade de extratos de Anadenanthera macrocarpa (Fabaceae: Mimosoideae) e Bougainvillea buttiana (Nyctaginaceae) para lepidópteros-praga. Biotemas, v. 30, n. 2, p. 15-24, junho de 2017.

9. CARVALHO, P. E. R. Espécies florestais brasileiras: recomendações silviculturais, potencialidades e uso da madeira. Brasília: EMBRAPA - SPI, 640 p. 1994.

10. CASTRO, A. S. Efeito de Morus nigra L. como terapia hormonal em ratas ooforectomizadas. Dissertação (Mestrado em Ciências da Saúde), Universidade Federal do Maranhão, São Luís, 2010.

11. CHEN, Y. et al. Morus alba and active compound oxyresveratrol exert anti-inflammatory activity via inhibition of leukocyte migration involving MEK/ERK signaling. BMC Complementary and Alternative Medicine, p.13:45. 2013.

12. DESMARCHELIER, C. et al. Antioxidant and free radical scavenging activities in extracts from medicinal trees used in the 'Caatinga' region in northeastern Brazil. Journal of Ethnopharmacology, v.67, p.69-77. 1999.

13. DIAS, T.G. Estudo farmacognóstico de Porangaba (Cordia ecalyculata Vell.boraginaceae) e identificação de adulterações. Dissertação de Mestrado, USP, São Paulo - SP, 2004.

14. DICKEL, O. E. et al. Efeitos comportamentais e neurotóxicos do extrato aquoso de Brugmansia suaveolens em ratos. Rev. Bras. Farm., v.91, n.4, p.189-99, 2010.

15. DUARTE, M.C.T. Atividade Antimicrobiana de Plantas Medicinais e Aromáticas Utilizadas no Brasil. Rev. MultiCiência: Construindo a História dos Produtos Naturais \#7, Out. 2006.

16. DUKE, J. A. Handbook of medicinal herbs. Flórida: CRC. Press, Inc., p. 21-420, 1985.

17. ERCISLI, S.; ORHAN, E. Chemical composition of white (Morus alba), red (Morus rubra) and black (Morus nigra) mulberry fruits. Food Chemistry, v.103, p.1380-1384, 2007.

18. FIGUEREDO, F.G. et al. Modulation of the Antibiotic Activity by Extracts from Amburana cearensis A. C. Smith and Anadenanthera macrocarpa (Benth.) Brenan. BioMed Research International.Volume 2013.

19. FREITAS, A.V.L. et al. Os raizeiros e a comercialização de plantas medicinais em São Miguel, Rio Grande do Norte, Brasil. R. bras. Bioci., Porto Alegre, v. 10, n. 2, p. 147-156, abr./jun. 2012.

20. KALANTARI, H.; AGHEL, N.; BAYATI, M. Hepatoprotective effect of Moru alba $L$. in carbon tetrachloride induced hepatotoxicity in micc. Saudi Pharm J. v.17, n.1, p.90-94. 2009.

21. KIM, E.S. et al. Purification and characterization of Moran 20K from Morus alba. Archives of Pharmaceutical Research, v. 22, p. 9-12, 1999.

22. LIMA, D. C. A.; CUNHA, G. O. S. Análise do perfil fitoquímico da toxicidade de Punica 
granatum e Morus nigra. VI Simpósio de Química, Educação para a Sustentabilidade, 2018.

23. LORENZI, H. Plantas medicinais no Brasil: nativas e exóticas cultivadas. Nova Odessa: Instituto Plantarum. p. 98. 2002.

24. LORENZI, H.; MATOS, F. J. A. Plantas medicinais no Brasil, nativas e exóticas. Instituto Plantarum. $2^{\circ}$ ed. Nova Odessa. São Paulo, 2008.

25. LÓS, D.W.S.; BARROS, R.P.; NEVES, J.D.S. Comercialização de Plantas Medicinais: Um Estudo Etnobotânico nas Feiras Livres do Município De Arapiraca-Al. Rev. De Biologia e Farmácia (BioFar). v. 7. N. 2. 2012.

26. LUNA, J.S., et al. A study of the larvicidal and molluscicidal activities of some medicinal plants from northeast Brazil. Journal of Ethnopharmacology, v.97, p.199-206. 2005.

27. MAIOLI-AZEVEDO, V.; FONSECA-KRUEL, V.S. Plantas medicinais e ritualísticas vendidas em feiras livres no Município do Rio de Janeiro, RJ, Brasil: estudo de caso nas zonas Norte e Sul. Acta bot. bras. V.21, n.2, p. 263275. 2007.

28. MATOS, F. J. A. O formulário fitoterápico do professor Dias da Rocha: informações sobre o emprego na medicina caseira, de plantas do Nordeste, especialmente do Ceará. 2. ed. Fortaleza: EUFC, 1997.

29. Meyer, B. N.et al. Brine Shrimp: A Convenient General Bioassay for ctive Plant Constituents. Journal of Medicinal Plant research. V. 45, p. 31-34. 1982.

30. MOHEBBI, A. et al.. Effect of Mulberry (Morus alba L.) Leaves Extract on the Secretion and Content of Triglyceride in the Chicken Hepatocytes Primary Culture. International Journal of Pharmacology. V. 3, p.116-119. 2007.

31. MONTEIRO, J. M. et al. Local Markets and Medicinal Plant Commerce: A Review with
Emphasis on Brazil. Economic Botany, v.64, n.4, p.352-356. 2010.

32. MUÑOZ, V. et al. The search for natural bioactive compounds through a multidisciplinary approach in Bolivia. Part II. Antimalarial activity of some plants used by Mosetene Indians. Journal of Ethnopharmacology. V. 69, p.139-155. 2000.

33. NOMURA, T. Phenolic compounds of the mulberry tree and related plants. Progress in the Chemistry of Organic Natural Produtcs, v. 53, p. 87-201, 1988.

34. NOMUERA, T.; HANO, Y. Isoprenoidsubstituted phenolic compounds of moraceous plants. Natural Products Reports, v. 11, p. 205 218, 1994.

35. OLIVEIRA, A.C.B. Avaliação toxicológica pré-clínica do chá das folhas de Morus nigra L. (Moraceae). Rev. Bras. Pl. Med., v. 15, n. 2, p. 244-249, 2013.

36. PARENTE, C. E. T.; ROSA, M. M. T. Plantas comercializadas como medicinais no Município de Barra do Pirai, RJ. Rodriguésia, v.52, n.80, p.47-59. 2001.

37. PADILHA, M.M. et al. Antinociceptive effect of Morus nigra leaves in mice. Journal of Medicinal Food, v. 12, n. 6, p. 1381-1385, 2009.

38. PELIZZA, M.C. Uso de Cereus sp. e Cordia ecalyculata Vell. como emagrecedores: uma revisão. Trabalho de Conclusão de Curso. UFRGS. Porto alegre - RS. 2010.

39. PIZZIOLO,V.R. et al. Plantas com possível atividade hipolipidêmica: uma revisão bibliográfica de livros editados no Brasil entre 1998 e 2008. Rev. Bras. Pl. Med., Botucatu, v.13, n.1, p.98-109, 2011.

40. RADI, P.A., TERRONES, M.G.H. Metabólitos secundários de plantas medicinais. Rev. Bras. Farm. V.20, n.2, p.18-22. 2007.

41. RODRIGUES, C. R. F., et al. Genotoxic and Antigenotoxic properties of Baccharis trime- 
ra in mice. Journal of Ethnopharmacology. V. 125, p.97-101. 2009.

42. RODRIGUES, L. A. et al. Elaboração de um fitoterápico antibiótico a partir da casca de Anadenanthera macrocarpa. Revista Científica Da Faminas - V. 10, N. 3, Set.-Dez. 2014.

43. SILVA, C.J.; BASTOS, J.K.; TAKASHI, C.S. Evaluation of the genotoxic and cytotoxic effects of crude extracts of Cordia ecalyculata and Echinodorus grandiflorus. Journal of Ethnopharmacology, v.127, p. 445-450. 2010.

44. SILVA, K. O. Avaliação das Atividades Antimicrobiana, Aderência, Antioxidante, Anti Inflamatória e Antinociceptiva de Anadenanthera Macrocarpa (Benth) Brenan. Dissertação de Mestrado. UFBA. Vitória da Conquista BA. 2011.

45. SIMÕES, C. et al. Farmacognosia da planta ao medicamento. $2^{\text {a }}$ ed. rev. Porto Alegre/ Florianópolis: Ed Universidade/UFRGS/ Ed. Universidade/ UFSC, 2000.

46. TEIXEIRA, L. N. et al. Screening Fitoquímico E Avaliação Do Potencial De Captura Do Radical Dpph Pelos Extratos De Manilkara sapota L. VII CONNEP. IFTO. 2012.

47. TÔRRES, A.R. et al. Estudo sobre o uso de plantas medicinais em crianças hospitalizadas da cidade de João Pessoa: riscos e benefícios. Revista Brasileira de Farmacognosia, v.15, n.4, p.373-380, 2005.

48. VALIATTI, T. B. et al. Estudo fitoquímico e análise das atividades citotóxica e antibacteriana das flores masculinas de carica sp. Revista da Universidade Vale do Rio Verde. V. 16, N. 2, ago./dez. 2018.

49. VENDRUSCOLO, G. S.; MENTZ, L. A. Levantamento etnobotânico das plantas utilizadas como medicinais por moradores do bairro Ponta Grossa, Porto Alegre, Rio Grande do Sul, Brasil. Iheringia, Sér. Bot., v. 61, n. 1-2, p. 83-103, 2006.

50. WANG, J. et al. Isolation of flavonoids from mulberry (Morus alba L.) leaves with macroporous resins. African Journal of Biotechnology, V. 7, n.13, p. 2147-2155, 4 July, 2008.

51. WASANO, N. et al. A unique latex protein, MLX56, defends mulberry trees from insects. Phytochemistry, v. 70, n. 7, p. 880-888, 2009.

52. YADAV, A.V.; NADE, V.S. Antidopaminergic effect of the methanolic extract of Morus alba L. leaves. Indian J Pharmacol. Oct; v.40, n.5, p.221-226. 2008.

53. ZHISHEN, J.; MENGCHENG, T.; JIANMING, W. A determinação dos teores de flavonoides na amoreira e seus efeitos de eliminação em radicais superóxidos. Química Alimentar, v.64, n.4, p.555-559, março de 1999.

\section{Richard da Silva Pereira Calazans}

Graduando em Farmácia pelo Centro Universitário Luterano de Ji-Paraná.

\begin{tabular}{l}
\hline Alexandra Luiza Silva Bulian \\
Graduanda em Farmácia pelo Centro Universitário \\
Luterano de Ji-Paraná. \\
\hline \\
\hline Lorraynie Oliveira Alves \\
Graduanda em Biomedicina pelo Centro \\
Universitário Luterano de Ji-Paraná.
\end{tabular}

Karine Amanda Costa

Graduanda em Farmácia pelo Centro Universitário Luterano de Ji-Paraná.

Jeferson de Oliveira Salvi
Mestre e Doutorando em Biologia Celular e
Molecular Aplicada a Saúde pela Universidade
Luterana do Brasil. Farmacêutico Especialista em
Acupuntura e Docente do Centro Universitário
Luterano de Ji-Paraná.

